\title{
EUV observations of CME-associated eruptive phenomena with the CORONAS-F/SPIRIT telescope/spectroheliograph
}

\author{
V.A. Slemzin ${ }^{1}$, V.V. Grechnev ${ }^{2}$, I.A. Zhitnik ${ }^{1}$, S.V. Kuzin ${ }^{1}$, \\ I.M. Chertok ${ }^{3}$, S.A. Bogachev ${ }^{1}$, A.P. Ignatiev ${ }^{1}$, A.A. Pertsov ${ }^{1}$ and \\ D.V. Lisin ${ }^{3}$ \\ ${ }^{1}$ P.N. Lebedev Physical Institute, Moscow, Russia, email: slem@sci.lebedev.ru \\ ${ }^{2}$ Institute of Solar Terrestrial Physics, Irkutsk, Russia, email: grechnev@iszf.irk.ru \\ ${ }^{3}$ IZMIRAN, Troitsk, Moscow Region, Russia, email: ichertok.izmiran.ru
}

\begin{abstract}
A multi-channel SPIRIT telescope/spectroheliograph aboard the CORONAS-F satellite operating in soft X-ray and EUV ranges $(T \sim 0.05-15 \mathrm{MK})$ is an effective instrument for complex studies of CME-associated phenomena such as eruptive filaments, dimmings, coronal waves, posteruptive arcades, etc. In particular, SRIRIT observations of high-temperature $(\mathrm{T}=$ 5-15 MK) plasma structures in the MgXII $8.42 \AA$ line show specific pre-CME sigmoid magnetic field configurations. Eruptions of filaments (prominences) and dimmings in a CME process are seen with a high contrast in the coronal $175 \AA$ band (FeIX-XI) and the transition-region $304 \AA$ (HeII) images. Our results are illustrated by several powerful eruptive events of the current solar cycle. We compare SPIRIT data with observations at other spaceborne and ground-based instruments (SOHO/EIT, Yohkoh/SXT, and $\mathrm{H} \alpha$ images, etc.)
\end{abstract}

Keywords. Sun: corona, Sun: coronal mass ejections (CMEs), Sun: X-rays, gamma rays

\section{Introduction}

CMEs are complex phenomena associated with filament eruptions and solar flares. They show a variety of kinematic and thermal transformations in the solar plasmas (Zhang et al. 2001; Moon et al. 2002; Feynman \& Ruzmaikin 2004). A pre-flare situation is often hinted by the presence of a sigmoidal loop system seen in hot ion lines of $\mathrm{T} \sim 2-$ $8 \mathrm{MK}$ (Gibson et al. 2002; DelZanna et al. 2002). To understand the origin and drivers of CMEs, important is to study the initial phase of the heating and acceleration of the erupting matter in the transition region and the corona at altitudes of several tenths $\mathrm{R}_{\odot}$, where it can be observed better in the EUV spectral range. Disappearance of a filament visible in the chromospheric $\mathrm{H} \alpha$ images is often followed by its appearance in transitionregion lines and, in some cases, in coronal lines later on. This stage of the CME process is not well studied so far, because necessary observations with sufficient time resolution simultaneously in several spectral lines usually are not available.

The SPIRIT experiment carried out aboard the CORONAS-F spacecraft since August 2001 (Zhitnik et al. 2002) provides synchronous observations of the whole Sun in several spectral bands (in particular, the transition-region band of $304 \AA$ and coronal bands of $175 \AA$ and $8.42 \AA$ ) covering the temperature range from 0.05 to $15 \mathrm{MK}$. Here we present some examples of SPIRIT observations of CME-related phenomena around the $23^{\text {rd }}$ 
solar maximum and compare it with observations at other spaceborne and ground-based instruments (SOHO/EIT, Yohkoh/SXT, H $\alpha$, etc.)

\section{SPIRIT observations and their comparison with data from other instruments}

The SPIRIT performs routine observations with an interval of 15 min during 45-min non-occulted period of the $93.5 \mathrm{~min}$ orbit. The observations are conducted simultaneously in two bands of the transition-region line $304 \AA$ (HeII, $T \sim 0.05-0.08 \mathrm{MK}$ ) and the coronal line $175 \AA$ (FeIX-XI, T 0.9-1.2 MK) with an exposure time of $2-9 \mathrm{~s}$. In comparison with the SOHO/EIT FeXII $195 \AA$ line, the $175 \AA$ band contains cooler lines of FeIX-XI with lesser dynamic range from the quiet Sun to flares. EIT images produced in $195 \AA$ line are often overexposured during flares and contaminated with strong scattered light, thus preventing observations of any features in the flaring region. By contrast, SPIRIT images remain clear.

The images in the MgXII $8.42 \AA$ line $(\mathrm{T} \sim 5-15 \mathrm{MK})$ are produced, as a rule, with an exposure time of $37 \mathrm{~s}$ and with an interval of 3-20 min. These images revealed hot coronal features with a typical peak temperature of $\sim 10 \mathrm{MK}$ and $\log N_{e} \sim 9-10$ (Zhitnik et al. 2003). They do not always coincide with active regions and significantly differ from lowertemperature (1-2 MK) structures seen in EUV coronal lines, such as 175 or $195 \AA$. These structures are often localized at very high altitudes of $0.1-0.3 R_{\odot}$ above the solar limb and live from minutes to days. They show fast spatial variability and have a contrast of more than two orders of magnitude.

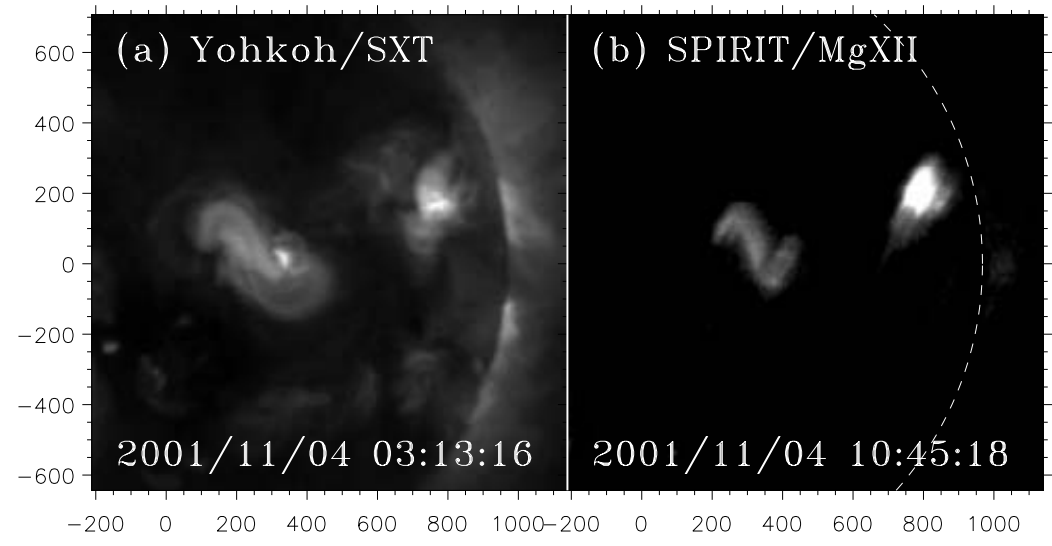

Figure 1. A 'sigmoid' in the flaring active region observed with Yohkoh/SXT and CORONAS-F/SPIRIT in MgXII line on November 4, 2001. White dashed line shows solar limb. Axes show arc seconds from solar disk center.

\section{SPIRIT observations of CME-related phenomena}

\subsection{Pre-event 'sigmoids' in MgXII images}

'Sigmoids' are believed to represent a highly sheared magnetic configuration overlying an extended filament channel (Glover et al. 2001) and to indicate a high probability of an eruption (Hudson et al. 1998). Sigmoids are clearly seen in soft X-rays, but poorly detectable in lower-temperature EUV emissions (Sterling et al. 2000). SPIRIT MgXII channel due to its high sensitivity to radiation of hot plasmas shows sigmoids with even 
better contrast than Yohkoh/SXT, indicating that the temperature of high pre-flare loops can exceed 5 MK. Fig. 1 shows a comparison of Yohkoh/SXT and SPIRIT/MgXII images for a powerful solar eruptive event of November 4, 2001 (Chertok et al. 2004). A sigmoid in AR 9684 preceded an X1.0 flare occurred at 16:03 UT and a fast (V $\sim 1800 \mathrm{~km} / \mathrm{s})$ halo CME at 16:35 UT. After the flare and CME, the loop structure transformed into a posteruptive arcade extending over $300000 \mathrm{~km}$ for more than one day.

\subsection{Posteruptive dimmings in SPIRIT $175 \AA$ and $304 \AA$ lines}

Coronal ('EIT') waves and dimmings are regarded as the most reliable signatures of a CME (see, e.g., Zhukov \& Auchère, 2004). Most often the coronal wave appears after a CME as a propagating bright front seen in coronal lines (e.g., EIT $195 \AA$ line). Behind the wave, a dimming appears as temporary, localized depletion of EUV radiation: some of them are deep and compact, other are shallower, but more extended, being directed to neighboring active regions. Possibly, some of extended dimmings are associated with large transequatorial loops (Farnik et al. 2001; Glover et al. 2003).

SPIRIT observations show many examples of coronal waves and a variety of dimmings, in particular, observed during the period of solar extreme events of October-November 2003 (Slemzin et al. 2004). Coronal waves were observed on October 26 and November 18 by the SPIRIT in $175 \AA$ and by the EIT in $195 \AA$. Dimmings observed for most powerful events of that period had very large scale to cover the hemisphere and showed conspicuous homology for many days, which suggests stability of the large-scale magnetic structure.
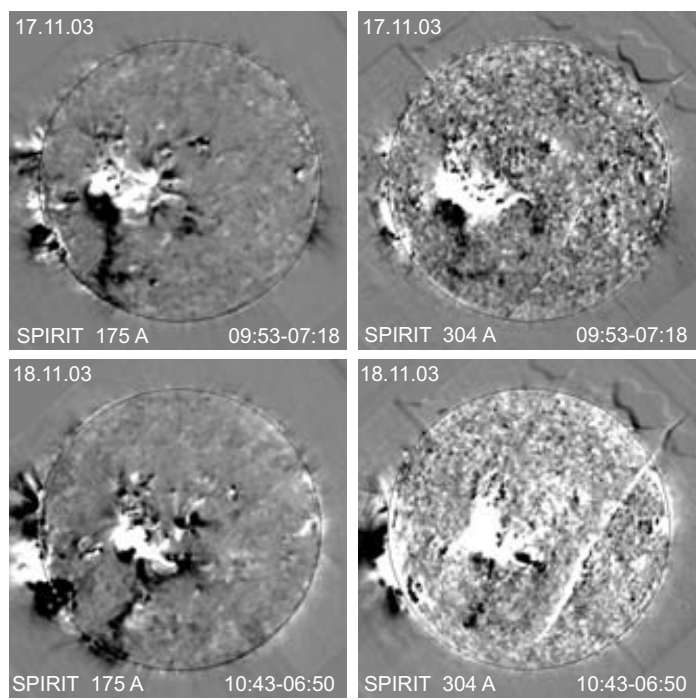

Figure 2. Structure of dimmings in CME events of November 17 and 18, 2003 in SPIRIT $175 \AA$ and $304 \AA$ channels

Fig. 2 shows a comparison of dimming structures observed on November 17 and 18, 2003 in the SPIRIT 175 and $304 \AA$ bands. It is clearly seen that the main dimmings are located near the eruptive center, the most intense of them is directed to the boundary of the southern polar coronal hole. The dimmings in both 175 and $304 \AA$ bands for two days are similar, but not fully coincide. A detailed analysis shows that they originated at the places of previous bright loop structures seen in the $175 \AA$ band before the events. The dimmings in the transition-region HeII $304 \AA$ line are similar in shape, but not coincide with those in $175 \AA$. As a rule, $304 \AA$ band dimmings have lesser depth and sometimes appear with significant delay of several tens minutes with respect to coronal 


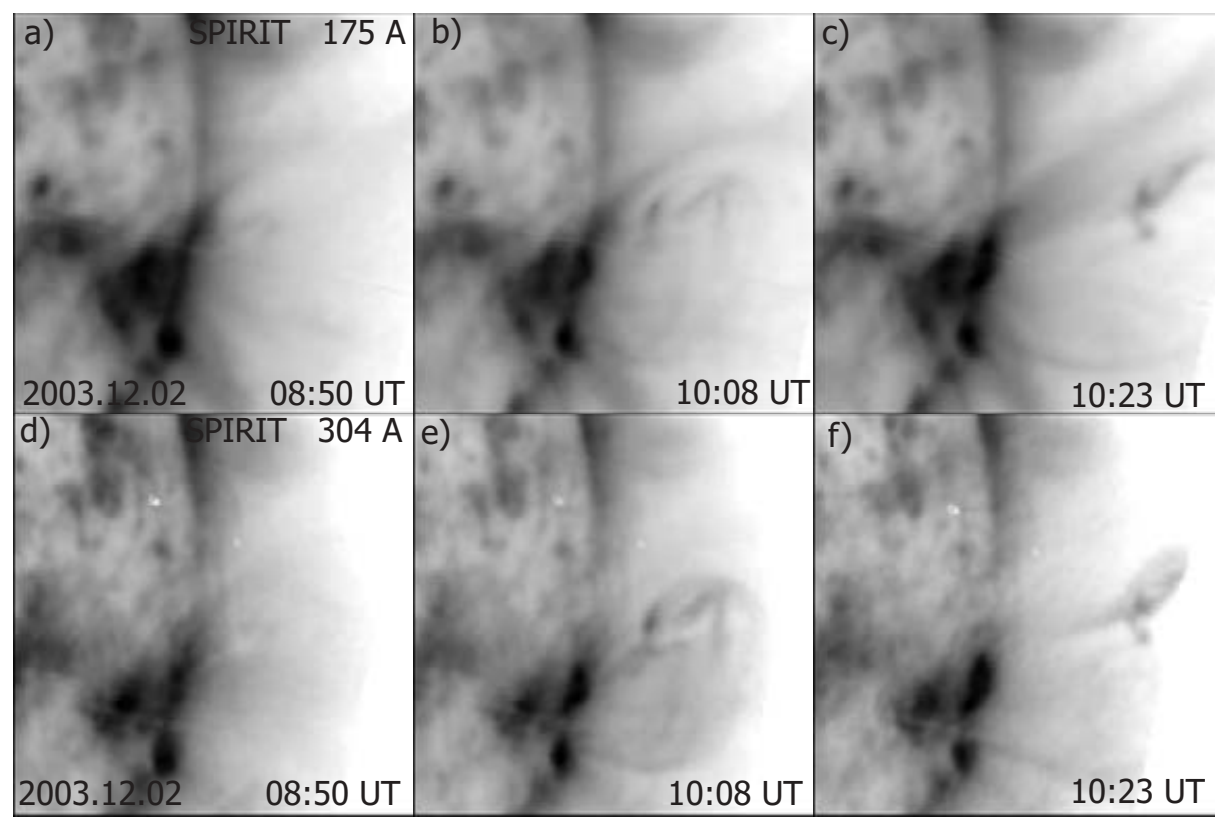

Figure 3. An eruptive event of December 2, 2003 in SPIRIT $175 \AA$ (a-c) and $304 \AA$ (d-f) bands (negative).

ones (Chertok et al. 2004). This probably suggests the development of the dimming from the higher coronal layers downward, up to the transition region.

\subsection{Eruptions of filaments and prominences}

Observations of filament (or prominence) eruptions are important to study the processes of CME formation and acceleration of eruptive mass. The temporal and spatial evolution of prominences is governed by dynamics of the magnetic field structure, but the trigger mechanism of eruption is still not well established (Schmieder et al. 2000, Marque et al. 2002). Simultaneous SPIRIT observations in $304 \AA$ and $175 \AA$ bands, due to their complementary temperature sensitivity ranges, are suitable to study plasmas in erupting structures at the initial stage of the CME launch.

We present two observations of eruptive events. In the first case, a fast ejection was observed after a prominence eruption on December 2, 2003 (fig. 3; Delaboudinière et.al 2005). A quiescent filament was observed during several days in $\mathrm{H} \alpha, 304 \AA$ band, and 175 \& $195 \AA$ coronal lines before the eruption, which occurred on December 2 at 10:15 UT. It produced a large CME with a speed of $\sim 1300 \mathrm{~km} / \mathrm{s}$. The eruptive prominence was observed first with Pic du Midi coronagraph in $\mathrm{H} \alpha$, then with EIT at $195 \AA$ and SPIRIT at $304 \& 175 \AA$ (fig. 3). The prominence becomes bright in both 175 and $195 \AA$ images, which implies its heating in the course of the eruption.

Another eruptive event was observed on June 8, 2004 during early Venus transit across the Sun (fig. 4). A large prominence slowly ascended (V $55 \mathrm{~km} / \mathrm{s})$ above the eastern limb for 40 min of observations. It was much brighter in $304 \AA$ line, than in the coronal $175 \AA$ line; hence, it was rather cold. It left the field of view of the SPIRIT at 05:41 UT at a height of $0.27 \mathrm{R}_{\odot}$, and the frontal structure of the corresponding CME appeared in LASCO/C2 image at 06:26 UT at $1.86 \mathrm{R}_{\odot}$. LASCO has not registered this event, because there were no subsequent $\mathrm{C} 2 / \mathrm{C} 3$ observations during several hours. 


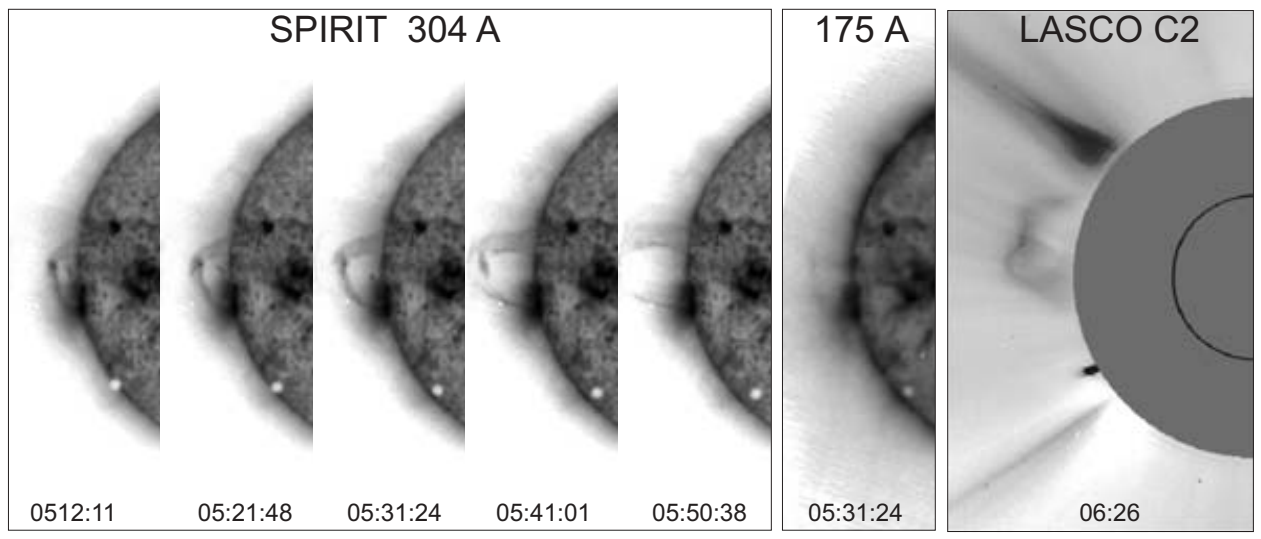

Figure 4. Eruption of a cold prominence during the Venus transit on June 8, 2004 (negative).

\section{Conclusions}

A multi-channel SPIRIT telescope/spectroheliograph operating aboard the CORONASF satellite allows studying various CME-associated phenomena in wide temperature range of $0.05-15 \mathrm{MK}$. The SPIRIT is able to record images in several spectral bands simultaneously with high cadence that is important to study dynamics of eruptive processes.

\section{Acknowledgements}

We are grateful to Prof. I.I. Sobelman and Prof. V.D. Kuznetsov, scientific leaders of the CORONAS-F project, for their interest and support of this study; A. Zhukov for fruitful discussions; and the CORONAS-F team in IZMIRAN for the assistance in the telemetry data supply. In this study we used SOHO data. SOHO is a project of international cooperation between ESA and NASA. This work is supported by the Russian Foundation for Basic Research (grants 02-02-17272, 03-02-16049, and 03-02-16591), the Federal Ministry of Industry and Science (grants NSh 477.2003.2 and 1445.2003.2), and the Program No. 16 of the General Physics Department of RAS.

\section{References}

Chertok, I.M. et al. 2004, Astronomy Rep. 48, 407.

Delaboudinière, J.-P., et al. 2005, this volume.

DelZanna, G., et al. 2002, Adv. Space Res. 30(3), 551.

Farnik, F. et al. 2001, Sol. Phys. 202, 81.

Feynman, J. \& A. Ruzmaikin 2004, Sol. Phys. 219, 301.

Gibson, S., et al. 2002, ApJ 574, 1021..

Glover, A., et al. 2001, $A \mathscr{E} A$ 378, 239.

Glover, A. et al. 2003, $A \& A A 400,759$.

Hudson, H.S. et al. 1998, Geoph. Res. Lett. 25(14), 2481.

Marque, C. et al. 2002, $A \mathscr{E} A 387,317$.

Moon, Y.-J., et al. 2002, $A p J 581,694$.

Sterling, A.C. et al. 2000, ApJ 532, 628.

Schmieder, B. et al. 2000, A\&\&A 358, 728.

Slemzin, V.A. et al. 2004, Proc. of IAUS 223 (in press).

Tripathi, D. et al. 2004, $A \& A 422,337$.

Zhang, J., et al. 2001, ApJ 559, 452.

Zhitnik, I.A. et al. 2002, ESA SP-506 915.

Zhukov, A. \& Auchère, F. 2004, A\& A 427, 705. 


\section{Discussion}

KAHLER: What is the time cadence of the SPIRIT images?

SLEMZIN: we have synoptic observations in several wavelengths at least once per $1.5 \mathrm{~h}$ orbit for 15 orbits a day. For some periods we take simultaneous images in $175 \AA / 304 \AA$ bands with a 15 min cadence during 45 min interval interrupted by 47-48 min occultations. We also participate in the "EIT Shutter-less" or "High cadence" program, when observations are done with a cadence less than 1 min.

P.F. Chen: Are the data available online?

SLEmzIn: We can provide on request. Some data can be found in MEDOC. Solar data center (France ), http://www. medoc.ias.u-psud.fr. 